ARTICLE TYPE

\title{
Regio- and Diastereoselective Pd-catalyzed Synthesis of C2-Aryl Glycosides
}

\author{
Juba Ghouilem, ${ }^{\mathrm{a}}$ Rémy Franco, ${ }^{\mathrm{a}}$ Pascal Retailleau, ${ }^{\mathrm{b}}$ Mouad Alami, ${ }^{\mathrm{a}}$ Vincent Gandon ${ }^{\mathrm{c}, \mathrm{d} *}$ and Samir \\ Messaoudi $^{\text {** }}$
}

An efficient regio- and diastereoselective arylation method of readily available 2,3-glycals with various aryl iodides has been established. Using the $\mathrm{Pd}(\mathrm{OAc})_{2} / \mathrm{AsPh}_{3}$ precatalytic 10 system, this protocol proved to be general to prepare a variety of substituted C2-aryl glycosides in good yields with complete diastereoselectivity.

C-aryl glycosides are key motifs in medicinal chemistry and drug discovery including antibiotics, antitumors, as well as antidiabetic

15 agents (Figure 1). ${ }^{1}$ These common motifs are also found in many natural bioactive compounds. ${ }^{1} \mathrm{C}$-aryl glycosides are considered as glycomimetics of biologically relevant $\mathrm{O} / \mathrm{N}$-glycosides and usually show enhanced resilience to enzymatic and hydrolytic cleavage under biological conditions. ${ }^{2}$ Thus, C-aryl glycosides 20 have emerged as a privileged class of saccharides with diverse potential applications. In this context, a plethora of methods ${ }^{3}$ related to the synthesis of C1-aryl glycosides has been reported, in which the aryl nucleus is linked to the sugar unit through the C-glycosidic bond. In contrast to this well-developed series, there 25 are still only few reports on the preparation of C2-aryl glycosides (Scheme 1). $\mathrm{C}-\mathrm{C}$ bond formation of $\mathrm{C}\left(\mathrm{sp}^{3}\right)$-hybridized glycosides suffers from a difficult installation of a reactive function at the $\mathrm{C} 2$ position of the sugar that could be further elaborated to furnish the targeted C2-aryl glycoside. Adding to this the difficulty to 30 control the stereoselectivity, C2-arylation is actually a very challenging synthetic endeavor.

Davis and co-workers reported a Suzuki cross-coupling approach $^{4}$ of 2-iodoglycals to furnish $\mathrm{C} 2\left(\mathrm{sp}^{2}\right)$-aryl glycosides in excellent yields (Scheme 1a). While this method highlights an 35 important achievement in this underdeveloped area, the narrow scope of only $O$-benzylated glycals and poor functional group tolerance limits its utilization. Recently, Mukherjee group

${ }^{a}$ Universite Paris-Saclay, CNRS, BioCIS, 92290, Châtenay-Malabry, France, Tel: + (33)0146835887; E-mail: samir.messaoudi@universite-paris-saclay.fr

${ }_{40}{ }^{b}$ Institut de Chimie des Substances Naturelles, CNRS UPR 2301, Universite ParisSaclay, avenue de la terrasse, 91198 Gif-sur-Yvette, France

Université Paris-Saclay, CNRS, ICMMO, 91405, Orsay cedex, France E-mail: vincent.gandon@universite-paris-saclay.fr

d Laboratoire de Chimie Moléculaire (LCM), CNRS UMR 9168, Ecole 45 Polytechnique, Institut Polytechnique de Paris, route de Saclay, 91128 Palaiseau cedex, France

+ Electronic Supplementary Information (ESI) available: General, experimental procedures for starting materials and ${ }^{1} \mathrm{H}$ and ${ }^{13} \mathrm{C}$ spectra for all new compounds. SeeDOI: $10.1039 / b 000000 x /$

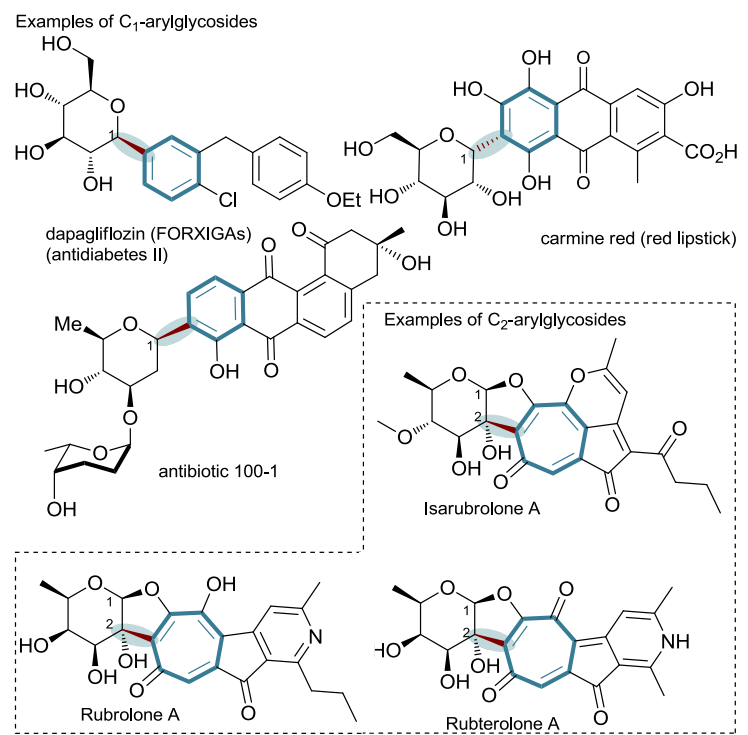

Figure 1. C-aryl glycosides-based bioactive molecules

reported an elegant method for the diarylation of glycals and pseudoglycals with aryl boronic acids under Pd-catalysis (Scheme $1 b) .{ }^{5}$ Selectivity was $\mathrm{C} 1-\mathrm{C} 2(\alpha, \alpha)$ in the case of glycals 55 but $\mathrm{C} 2-\mathrm{C} 3(\beta, \beta)$ for pseudoglycals. However, this method is limited to acetylated glycals and only leads to symmetrical diarylated C-glycosides. Another way to prepare C2-aryl glycosides reported by Tenaglia et al., ${ }^{6}$ consists in the transformation of $o$-iodoaryl branched hex-2-enopyranosides 60 through an intramolecular Heck reaction to cis-fused aryl pyranosides (Scheme 1c). Based on these few literature reports to access to C2-aryl glycosides, we became interested in developing a direct approach. In this context, we reported recently an efficient protocol for the synthesis of functionalized C2-aryl ${ }_{65}$ glycosides via a $\mathrm{Pd}(\mathrm{II})$-catalyzed diastereoselective $\mathrm{C}\left(\mathrm{sp}^{3}\right)-\mathrm{H}$ activation approach using a proximity-driven metalation strategy (Figure 1d). ${ }^{7}$ This method exhibits a 2,3-trans-arylation selectivity and tolerates a wide range of functional groups. Despite the substantial improvement of this approach, the 70 stoichiometric installation and removal of the directing group is time consuming and generates wastes, which limits its efficiency and applicability. To avoid these additional steps, we decided to pursue our investigations in developing direct and diastereoselective methods for the synthesis of C2-aryl 75 glycosides. In this context, we became interested in the reactivity of 2,3-pseudoglucals. Although these substrates are easily accessible in a single step from commercially available glycals, 


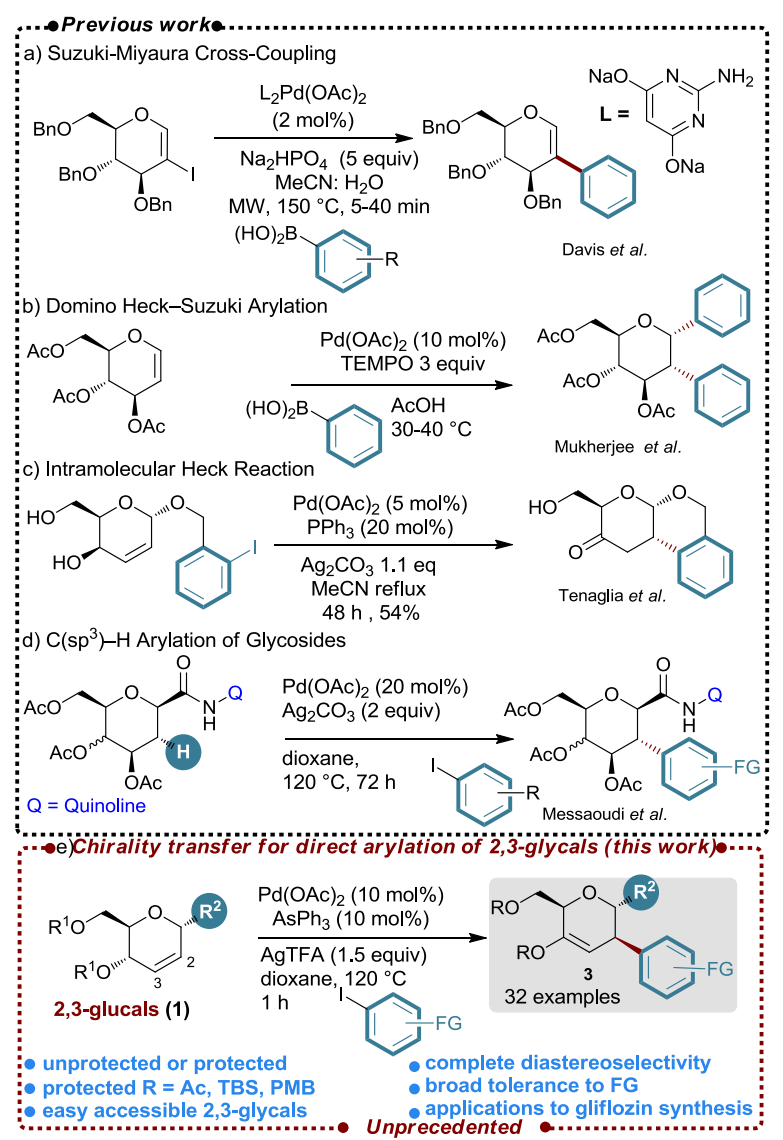

Scheme 1. Strategies to access C2-aryl glycosides.

only few studies report their functionalization. We envisaged that the C2-diastereoselective introduction of an aryl moiety on these 5 substrates could be accomplished through a Mizoroki-Heck reaction (Scheme 1e). We hypothesized at this stage that the arylpalladium $^{(\mathrm{II})}$ complex will approach the double bond of pseudoglucal at the opposite face of the $\mathrm{C}_{3}-\mathrm{OR}$ bond (Figure 2). This pathway will lead to complex (B), which possesses $\mathrm{H} 4$ as 10 the only hydrogen that may be involved in a syn- $\beta$-elimination process. We anticipated that the stereochemistry at the $\mathrm{C} 2$ position of the coupling product would be dictated by the stereochemistry at the $\mathrm{C} 4$ position of the pseudoglucal through a chirality transfer mechanism. Thus, through this work, we show 15 for the first time, that pseudoglucals can be successfully arylated

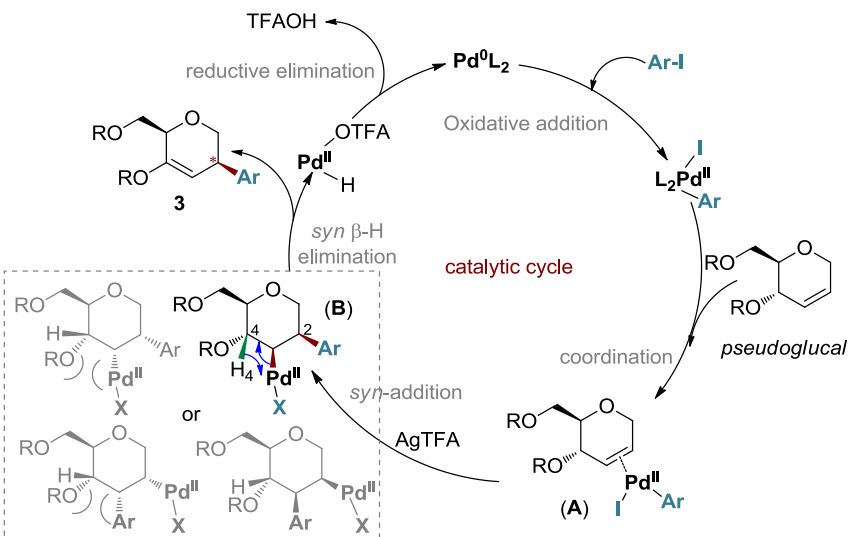

Figure 2. Proposed mechanism for the Mizoroki-Heck C2-arylation of pseudoglucals. by a Mizoroki-Heck process to afford in a single step a variety of 20 substituted $\mathrm{C} 2$-aryl glycosides in a diastereoselective fashion (Figure 1e). Interestingly, we evidenced during this study the crucial directing effect of the $\mathrm{C}_{4}-\mathrm{OR}$ substituent to control the regio- and the stereoselectivity.

To initiate this study, we conducted the coupling of acetylated 25 2,3-pseudoglucal 1a with 4-iodomethylbenzoate $\mathbf{2 a}$ under various reaction conditions. Representative results are summarized in Table 1 (see the ESI for the full optimization). In preliminary experiments, the Heck reaction was first examined in the presence of $\mathrm{Pd}(\mathrm{OAc})_{2}$ as a pre-catalyst (10 $\left.\mathrm{mol} \%\right), \mathrm{PPh}_{3}(20$ $30 \mathrm{~mol} \%$ ) as ligand and $\mathrm{Ag}_{2} \mathrm{CO}_{3}$ as a base in acetonitrile at $120{ }^{\circ} \mathrm{C}$ for $36 \mathrm{~h}$. Under these conditions, we observed $63 \%$ conversion of the starting pseudoglucal and only $37 \%$ of isolated yield of the desired product, as a single diastereoisomer (entry 2). 1D and 2D NMR analyses clearly showed the 2,5-cis configuration and 35 demonstrated the high diastereoselectivity of this reaction. After screening several parameters (Table 1 or ESI), we finally found that the C2-arylation occurred smoothly with $76 \%$ yield when using $\mathrm{Pd}(\mathrm{OAc})_{2}$ as a pre-catalyst $(10 \mathrm{~mol} \%), \mathrm{AsPh}_{3}(10 \mathrm{~mol} \%)$ as ligand and AgTFA as a base in dioxane at $120{ }^{\circ} \mathrm{C}$ for $1 \mathrm{~h}$. 40 Interestingly, in all conditions examined during this optimization step, only one diastereoisomer 3a was isolated. It should be noted that the $\mathrm{Pd}(\mathrm{OAc})_{2}$ and $\mathrm{AsPh}_{3}$ were necessary to achieve this transformation since 3a was not observed when the coupling was conducted in their absence.

45 Table 1 Survey of the conditions for the C2-arylation of $1 \mathbf{a}$ with $\mathbf{2} \mathbf{a}^{\mathrm{a}}$

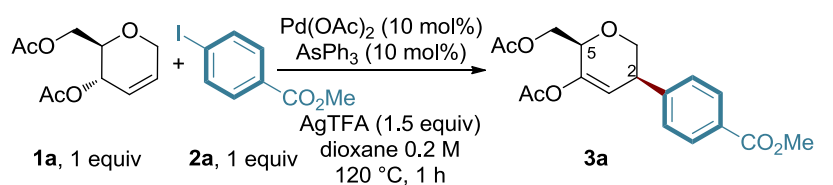

\begin{tabular}{lcl}
\hline Entry & Deviation from the standard conditions & Yield $(\%)^{b}$ \\
\hline 1 & none & 76 \\
2 & $\mathrm{PPh}_{3}(20 \mathrm{~mol} \%), \mathrm{Ag}_{2} \mathrm{CO}_{3}$ in $\mathrm{MeCN}$ for $36 \mathrm{~h}$ & 37 \\
3 & 2.5 equiv of $\mathrm{AgTFA}$ & 80 \\
4 & $5 \mathrm{~mol} \%$ of Pd cat. and $7.5 \mathrm{~mol} \%$ of $\mathrm{AsPh}_{3}$ & 70 \\
5 & DMF instead of dioxane & 33 \\
6 & CPME instead of dioxane & 27 \\
7 & Toluene instead of dioxane & 32
\end{tabular}

Reactions were conducted with substrate 1a $(0.4 \mathrm{mmol}), \mathbf{2 a}(0.4 \mathrm{mmol})$, $\mathrm{Pd}(\mathrm{OAc})_{2}(0.04 \mathrm{mmol})$, additive, and solvent $(2.0 \mathrm{~mL}) .{ }^{b}$ Yield of isolated product 3a.

50 Motivated by these results, we next explored the scope of the direct C2-arylation of various pseudoglycals 1a-h with aryl iodides. At first, we were pleased to see that various aryl iodides bearing diverse functions $\left(-\mathrm{CO}_{2} \mathrm{Me},-\mathrm{CHO},-\mathrm{COMe},-\mathrm{Cl},-\mathrm{F}\right.$, $\mathrm{Br},-\mathrm{I},-\mathrm{CF}_{3},-\mathrm{CN},-\mathrm{NO}_{2},-\mathrm{OAc}$ and $-\mathrm{OMe}$ ) reacted smoothly 55 with 1a to afford the desired C2-aryl glycosides 3a-w in moderate to good yields and high diasteroselectivity (Scheme 2). The exact structure of 3n, including its relative 2,5-cis geometry, was unambiguously confirmed by crystal structure analysis. Of note, the presence of an ortho substituent at the aromatic ring of the 60 coupling partner did not affect the reaction process, as compounds $\mathbf{3 k}$, 3l, $\mathbf{3 s}$ and $\mathbf{3 t}$ were obtained in satisfactory yields. Finally, the synthetic utility of this methodology was 

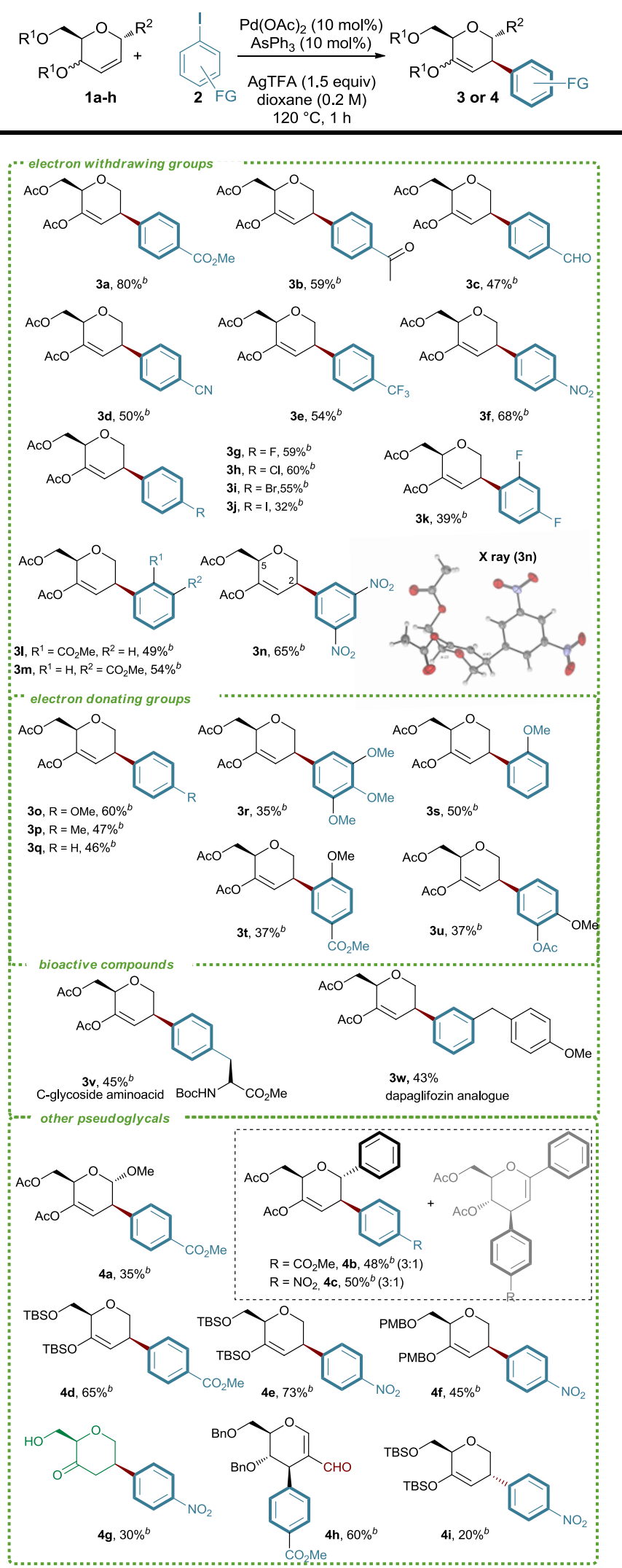

Scheme 2. Scope of the coupling of $\mathbf{1 a - h}$ with various aryl iodides $\mathbf{2}^{a}$

${ }^{a}$ Reactions were performed in a flame dried re-sealable Schlenk tube using pseudoglucals 1a-h $(0.4 \mathrm{mmol})$, ArI $(0.4 \mathrm{mmol}), \mathrm{Pd}(\mathrm{OAc})_{2}(0.04$

$5 \mathrm{mmol}), \mathrm{AsPh}_{3}(0.04 \mathrm{mmol}), \operatorname{AgTFA}(0.6 \mathrm{mmol})$ in dioxane $(2.0 \mathrm{~mL})$ at $120{ }^{\circ} \mathrm{C} .{ }^{b}$ Yield of isolated product. demonstrated by the synthesis of the C-glycosyl amino acid analogue $3 \mathbf{v}$ through the coupling of $p$-iodo phenylalanine with 1a. This finding holds major potential for the preparation of $C$ 10 aryl glycosyl amino acid building blocks for their subsequent use in glycopeptide assembly. In addition, C-glycoside 3w, an analogue of the dapagliflozin drug (FORXIGAs) ${ }^{8}$ used to treat type 2 diabetes (Figure 1) was successfully synthesized in $43 \%$ yield by this methodology. Next, we moved on to investigate the 15 reactivity of a series of pseudoglycals $\mathbf{1 b}$-h. As depicted in Table 2 , this coupling reaction tolerates different substituents at the $\mathrm{C} 1$ position of pseudoglucals such as $-\mathrm{OMe}$ (product 4a) or $-\mathrm{Ph}$ (compounds 4b-c). Noteworthy, in the case of C-glycosides $\mathbf{4 b}$ and $\mathbf{4 c}$, a mixture of separated regioisomers was obtained in a 3:1 20 ratio. In addition, this methodology tolerates other protecting groups such -OTBS (compounds 4d-e) and -PMB (compound 4f). Interestingly, the reaction tolerates unprotected pseudoglucals such as the C-glycoside 4g. One can note that when the benzylated pseudoglucal $\mathbf{1 g}$ bearing a formyl group at the $\mathrm{C} 2$ 25 position was used, inverted reactivity was observed in favor the C3-aryl glycoside $4 \mathbf{h}$, which was obtained in $60 \%$ yield as a single stereoisomer. Finally, to confirm that the diastereoselectivity of this coupling was driven by the configuration at the $\mathrm{C} 4$-position, we performed the reaction with 30 pseudogalactal $\mathbf{1 h}$ instead of $\mathbf{1 a}$. Interestingly, the expected product $\mathbf{4 i}$, in which the $\mathrm{C} 2$-configuration was inverted $(2,5$ trans), was obtained. However, the yield did not exceed $20 \%$.

DFT computations were performed to rationalize the regio- and the diastereoselectivity observed, focusing on the insertion step 35 (Figure 3). Pseudoglucal 1a was used as substrate. Two possible pathways were envisioned for the syn arylpalladation of this compound (Figure 3): i) a cationic mechanism involving [Ph$\left.\mathrm{Pd}\left(\mathrm{AsPh}_{3}\right)\right]^{+} ;$ii) a neutral mechanism involving $[\mathrm{Ph}-$ $\left.\operatorname{Pd}(\mathrm{TFA})\left(\mathrm{AsPh}_{3}\right)\right]$. Coordination of palladium to the substrate 40 leads to intermediates of type Int-cis or Int-trans, in which the metal fragment is in a cis- or trans-relationship with the substituent at C5. Int-C2-cis has been taken as reference complex for the free energies $(0.0 \mathrm{kcal} / \mathrm{mol})$. From Int-cis and Int-trans intermediates, arylation at $\mathrm{C} 2$ and $\mathrm{C} 3$ has been computed.

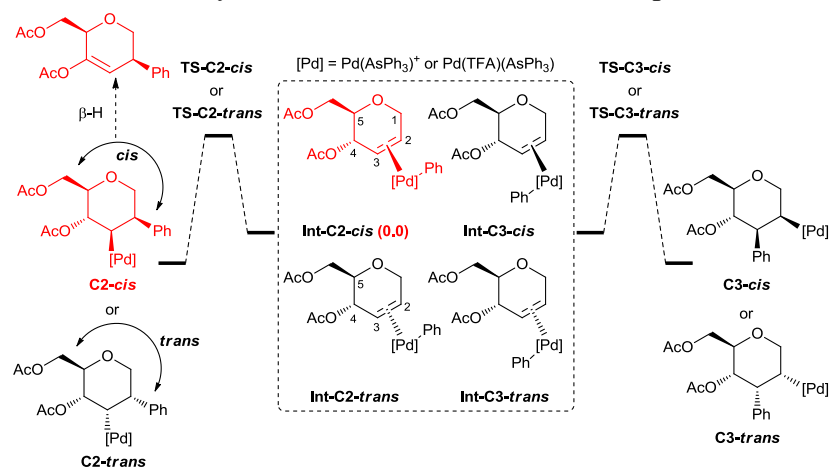

Figure 3. Free energy profile of the syn-insertion pathways.

Of note, only $\mathbf{C} 2$-cis can lead to the experimentally observed product after syn $\beta-\mathrm{H}$ elimination and reductive elimination. The free energies related to Figure 3 were calculated and are reported 50 in Table 2 (see also Figures S2 and S3 in the ESI). Complexes of type $\mathbf{C} 2$-trans cannot give rise to a $\beta-\mathrm{H}$ elimination and should 
therefore be considered as unproductive. They were included nonetheless for comparison. On the basis of the free energy of activations, it seems clear that the fastest reaction is the one corresponding to the formation of $\mathbf{C} 2$-cis. In the cationic series, 5 the barrier of $15.3 \mathrm{kcal} / \mathrm{mol}$ is markedly lower than those leading to the other isomers $(22.6-24.3 \mathrm{kcal} / \mathrm{mol})$. In the neutral series, the barrier of $15.0 \mathrm{kcal} / \mathrm{mol}$ is $1.2 \mathrm{kcal} / \mathrm{mol}$ lower than the one leading to $\mathbf{C} 3$-cis $(16.2 \mathrm{kcal} / \mathrm{mol})$. The formation of the trans isomers is significantly more demanding in free energy of 10 activation ( 18.1 and $17.4 \mathrm{kcal} / \mathrm{mol})$. In the two series, the formation of C2-cis is also more exergonic (-10.4 and -6.5 $\mathrm{kcal} / \mathrm{mol}$ for the cationic and neutral pathways respectively). Although it is difficult to make a clear cut between the cationic and the neutral pathways, any of the two favors the arylation at ${ }_{15} \mathrm{C} 2$ and place the aryl group in the cis-relationship with the $\mathrm{CH}_{2} \mathrm{OAc}$ substituent at $\mathrm{C} 5$.

Table 2. Free energies $\left(\Delta G_{393}, \mathrm{kcal} / \mathrm{mol}\right)$ of the computed intermediates and transition states referenced to Int-C2-cis

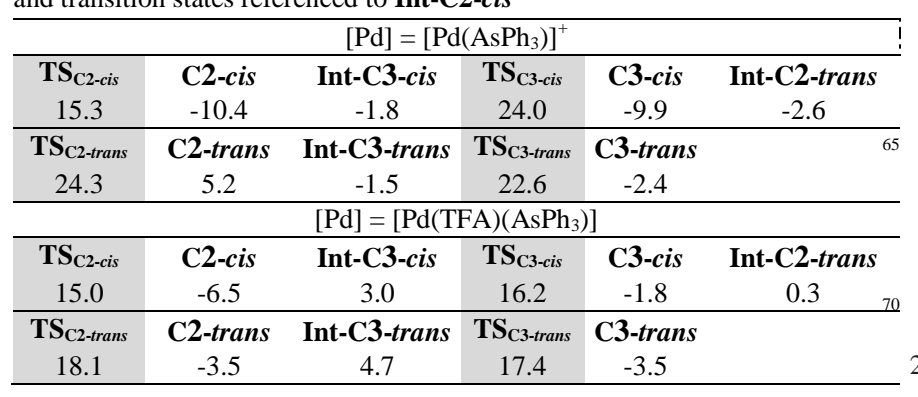

The geometries corresponding to the $\mathbf{C} 2$-cis series are collected 20 in Figure S1 of the ESI. With the cationic catalyst, a strong interaction between the acetate at $\mathrm{C} 4$ and palladium is clearly revealed. It leads to a $\eta^{1}$-coodination of $\mathrm{Pd}$ to the $\mathrm{C}=\mathrm{C}$ bond of the substrate and engage the metal into a 6-membered ring system. This $\mathrm{Pd}-\mathrm{O}$ interaction is still present in the transition state 25 (Figure 4) and in the product (ESI). Interestingly, this type of control is reminiscent to DG-arylation, with the acetates thus being crucial to control the regio- and the stereoselectivity. Such an electronic directing effect is in principle also possible from Int-C3-trans. However, in this case, it does not involve the 30 carbonyl oxygen but the one directly connected to the glucal ring. It is therefore less efficient as the resulting metallacycle is a 4membered ring instead of a 6-membered one. On the other hand, the rationalization of the regio- and diastereochemistry of the neutral pathway can be made on the basis of steric effects. The 35 metal fragment prefers the most accessible side of the $\mathrm{C}=\mathrm{C}$ bond, i.e. anti to the $\mathrm{OAc}$ group at $\mathrm{C} 4$. The $\mathrm{Ph}$ group is oriented towards $\mathrm{C} 2$, which is a $\mathrm{CH}_{2}$, rather than $\mathrm{C} 3$, to limit the steric demand imposed by $\mathrm{C} 4$, which is trisubstituted.
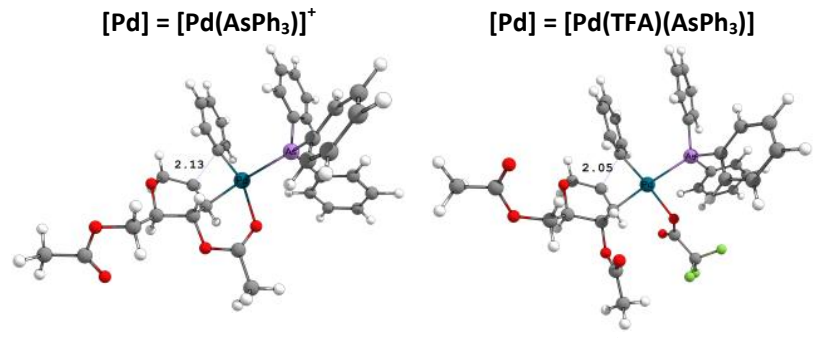

Figure 4. Geometries of $\mathbf{T S}_{\mathbf{C 2} \text {-cis }}$ (selected distances in $\AA$ ).

40 In conclusion, we successfully developed an efficient and diastereoselective method for the synthesis of C2-aryl glycosides by coupling 2,3-pseudoglucals with aryl iodides under palladium catalysis. The protocol exhibits a broad substrate scope with respect to the coupling partners, thus providing an attractive 45 access to a large molecular diversity of C2-substituted glycosides. Moreover, DFT calculations were performed to elucidate the unexpected 2,5-cis diastereoselectivity of this reaction.

\section{Acknowledgements}

Authors acknowledge support of this project by CNRS, Universite Paris50 Saclay, ANR (SelFSuCHi, ANR-18-CE07-0012), Ecole Polytechnique, la Ligue Contre le Cancer through an Equipe Labellisée 2014 grant. Our laboratory is a member of the Laboratory of Excellence LERMIT supported by a grant (ANR-10-LABX-33).

\section{Notes and references}

55 1. For review, see: a) A. Dondoni and A. Marra, Chem. Rev., 2000, 100, 4395; b) É. Bokor, S. Kun, D. Goyard, M. Toth, J.-P. Praly, S. Vidal and L. Somsaḱ, Chem. Rev., 2017, 117, 1687; c) Y. Yang and B. Yu, Chem. Rev., 2017, 117, 12281; d) D. C. Koester, A. Holkenbrink and D. B. Werz, Synthesis, 2010, 19, 3217; e) Z. Wei, Curr. Top. Med. Chem., 2005, 5, 1363; For selected publications, see: f) G. Yang, J. Schmieg, M. Tsuji and R. W. Franck, Angew. Chem. Int. Ed., 2004, 43, 3818; g) R. W. Franck and M. Tsuji, Acc. Chem. Res., 2006, 39, 692; h) H. Liao, J. Ma, H. Yao and X.-W. Liu, Org. Biomol. Chem., 2018, 16, 1791; i) K. Krohn, A. Agocs and C. Bäuerlein, J. Carbohydr. Chem., 2003, 22, 579; j) D. Y. W. Lee, W. Y. Zhang and V. V. R. Karnati, Tetrahedron Lett., 2003, 44, 6857; k) M. A. Ali and L. Haynes, J. Chem. Soc., 1959, 1033; 1) T. Cañeque, F. Gomes, T. Mai, G. Maestri, M. Malacria and R. Rodriguez, Nature Chem., 2015, 7, 744; m) Y. Yan, J. Yang, L. Wang, D. Xu, Z. Yu, X. Guo, G. P. Horsman, S. Lin, M. Tao, and S.-X. Huang, Chem. Sci., 2020, 11, 3959.

2. a) E. C. Chao and R. R. Henry, Nat. Rev. Drug Discovery, 2010, 9, 551; b) X. J. Wang, L. Zhang, D. Byrne, L. Nummy, D. Weber, D. Krishnamurthy, N. Yee, C. H. Senanayake, Org. Lett., 2014, 16, 4090; c) J. P. Henschke, C. W. Lin, P. Y. Wu, W. S.Tsao, J. H. Liao and P. C. Chiang, J. Org. Chem., 2015, 80, 5189; d) C. Guo, M. Hu, R. J. Deorazio, A. Usyatinsky and K. Fitzpatrick, Z. Zhang, J. H. Maeng, D. B. Kitchen, S. Tom, M. Luche, Bioorg. Med. Chem., 2014, 22, 3414.

80 3. a) X. Li and J. Zhu, Eur. J. Org. Chem., 2016, 2016, 4724; b) Y. Dai, B. Tian, H. Chen and Q. Zhang, ACS Catal., 2019, 9, 2909; c) J. Liu and H. Gong, Org. Lett., 2018, 20, 7991; e) F. Zhu, J. Rodriguez, S. O'Neill and M. A. Walczak, ACS Cent. Sci., 2018, 4, 1652; d) F. Zhu, J. Rodriguez, T. Yang, I. Kevlishvili, E. Miller, D. Yi, S. O'Neill, M. J. Rourke, P. Liu and M. A. Walczak, J. Am. Chem. Soc., 2017, 139, 17908; e) L. Adak, S. Kawamura, G. Toma, T. Takenaka, K. Isozaki, H. Takaya, A. Orita, H. C. Li, T. K. M. Shing and M. Nakamura, J. Am. Chem. Soc., 2017, 139, 10693; f) F. Zhu, M. J. Rourke, T. Yang, J. Rodriguez and M. A. Walczak, $J$. $90 \quad$ Am. Chem. Soc., 2016, 138, 12049; g) J. Zeng, J. Ma, S. Xiang, S. Cai and X.-W. Liu, Angew. Chem. Int. Ed., 2013, 52, 5134; h) L. Nicolas, P. Angibaud, I. Stansfield, P. Bonnet, L. Meerpoel, S. Reymond and J. Cossy, Angew. Chem. Int. Ed., 2012, 51, 11101; i) H. Gong and M. R. Gagné, J. Am. Chem. Soc., 2008, 130, 12177; j) M. Liu, Y. Niu, Y.-F. Wu and X.-S. Ye, Org. Lett., 2016, 18, 1836; 1) X. Li and J. Zhu. Eur. J. Org. Chem., 2016, 4, 724; 1) T. Mabit, A. Siard, F. Legros, S. Guillarme, A. Martel, J. Lebreton, F. Carreaux, G. Dujardin and S. Collet, Chem. Eur. J., 2018, 24, 14069; m) Q. Wang, S. An, Z. Deng, W. Zhu, Z. Huang, G. He and G. Chen, Nat. Catal., 2019, $2,793$.

4. I. Cobo, M. Matheu, S. Castillón, O. Boutureira, and B. G. Davis, Org. Lett., 2012, 14, 7, 1728.

5. A. Kusunuru, C. Jaladanki, M. Tatina, P. Bharatam and D. Mukherjee. Org. Lett., 2015, 17, 15, 3742.

$1056 . \quad$ A. Tenaglia and F. Karl. Synlett., 1996, 4, 327.

7. N. Probst, G. Grelier, S. Dahaoui, M. Alami, V. Gandon and S. Messaoudi, ACS Catal., 2018, 8, 9, 7781.

8. W. Cai, L. Jiang, Y. Xie, Y. Liu, W. Liu and G. Zhao, Med. Chem., $2015,11,317$. 